without the formation of any cyst, when the free water completely disappeared from the agar surface. This phenomenon appears to be comparable with the condition of anabiosis shown by Tardigrada and other animalcules of the moss fauna, and so far as I have been able to discover, it has not previously been reported for any ciliate. In this condition the body of the ciliate has a shrivelled and flattened appearance and shows no signs of life. It is, however, capable of revival within periods of up to three weeks, provided that it is in contact with some moisture-retaining substance, and that the relative humidity is high. Immediately upon the addition of water, the cilia commence to beat, the organism starts to swim about, and in a period varying from ten minutes to an hour it has regained its normal form and activity.

Balantiophorus minutus is thus peculiarly well fitted for life in the surface soil, where the amount of free water is subject to frequent and drastic alteration, and is at all times limited save in water-logged soils. It is not unlikely that upon further investigation other soil ciliates will be found to possess a similar power of temporary resistance to desiccation.

A fuller account of this work is in the course of preparation, and it is hoped that it will appear shortly.

In conclusion, it may not be out of place to remark that Balantiophorus minutus shows a similar ability to cause flocculation of the bacteria on which it feeds to that reported in these columns for Oikomonas termo by Hardin ${ }^{1}$ and for Epistylis and Vorticella by Pillai and Subrahmanyan ${ }^{2}$.

Wellcome Bureau of Scientific Research, JoHN M. WATSON. 183-193 Euston Road, London, N.W.1.

I NTERR, 151, 642 (1943)

2 NATURR, 150, 525 (1942).

\section{Hitherto Unidentified Absorption Bands of Water Vapour}

Is 1912-13, F. E. Fowle ${ }^{1}$ made studies of the absorption of infra-red radiation by water-vapour at $0.93 \mu, 1.13 \mu$ and $1.47 \mu$, in order, to determine spectroscopically the total water-vapour content of the atmosphere. The first two wave-lengths are at the centres of the $\rho$ and $\varphi$ bands; the last is about $450 \mathrm{~cm} .^{-1}$ from the $\psi$-band centre.

Recently, studying the rotation-band of watervapour numerically, I found a striking difference in absorption near the band-centre and at the wing. Assuming a line-width $0.4 \mathrm{~cm} .^{-1}$, I found that, to increase the absorption from 20 to 80 per cent, a 25-fold increase in the quantity of absorbing vapour is needed near the band-centre, but a 40 -fold increase is needed near the wing. Fowle's results indicate a 25 -fold increase at each of the three wave-lengths. This suggests that the absorption at $1 \cdot 47 \mu$ may be due to a hitherto unidentified band, centred near $1 \cdot 47 \mu$. It is, in any event, improbable that the $\psi$-band should give absorption more than $450 \mathrm{~cm} .^{-1}$ from the band-centre, when the much stronger band centred at $6 \cdot 3 \mu$ extends, on the long-wave side, only $400 \mathrm{~cm}^{-1}$ from its centre.

Mecke's empirical formula ${ }^{2}$ suggests that the new band is the band $\nu_{3}+2 \nu_{2}\left(\nu_{\sigma}+2 \delta_{\pi}\right)$, centred about $1 \cdot 455 \mu$. To test this, energy-levels for $v_{3}+2 v_{2}$ were estimated roughly by extrapolation from Nielsen's data $^{3}$ for $\nu_{2}, 2 \nu_{2}, \nu_{3}, \nu_{3}+\nu_{2}$, and those of Randall, Dennison, Ginsburg and Weber" for the rotation- band. These gave rough positions for the important lines in the band, which were found to be about $5 \mathrm{~cm} .^{-1}$ below the positions of lines observed in sunlight by Abbott and Freeman ${ }^{5}$. The computed energy-levels were increased by about this amount, when it was found that all strong lines predicted between $1.44 \mu$ and $1 \cdot 48 \mu$ were actually observed, and no others. This establishes the correctness of the identification. The lowest energy-levels are tabulated below.

$\begin{array}{llllll}0_{0} & 6876 \mathrm{~cm} .^{-1} & 2_{\theta} & 6978 \mathrm{~cm}^{-1} & 3_{0} & 7096 \mathrm{~cm} .^{-1} \\ 1_{2} & 6922 & 2_{-1} & 6961 & 3_{-1} & 7055 \\ 1_{0} & 6918 & 2_{-3} & 6945 & 3_{-2} & 7022 \\ 1_{-1} & 6899 & 3_{3} & 7222 & 3_{-y} & 7019 \\ 2_{2} & 7031 & 3_{2} & 7221 & & \\ 2_{1} & 7030 & 3_{1} & 7113 & & \end{array}$

The new band has evaded detection so far because of its weakness, which makes it hard to observe in the laboratory, and its proximity to the $\psi$-band. Other weak bands can, probably, also be identified in sunlight. The lines observed by Langley ${ }^{6}$ at $2 \cdot 115 \mu$ and $2 \cdot 1645 \mu$ are probably the maxima of the two wings of the 'butterfly' band $3 v_{2}$, and the lines found by Abbott and Freeman ${ }^{5}$ between 1.178 $\mu$ and $1 \cdot 21 \mu$ probably arise from the band $\nu_{3}+3 v_{2}$, centred near $1 \cdot 192 \mu$. Each of these bands is, however, overlapped by stronger bands, which have hitherto masked their presence.

Department of Mathematics,

T. G. Cowling.

University, Manchester.

$$
\text { Oct. } 29 .
$$

1 Fowle, F. E., Astrophys. J., 35, 149 (1912); 37, 359 (1913).

${ }^{2}$ Mecke, R., Z. Phys., 81, 313 (1933).

${ }^{3}$ Nielsen, H. H., Phys. Rev., 59, 565 (1941); 62, 422 (1942).

${ }^{4}$ Randall, H. M., Dennison, D. M., Ginsburg, N., and Weber, L. R., Phys. Rev., 52, 160 (1937).

s Abbott, C. G., and Freeman, H. B., Smithson. Misc. Coll., 82, No. 1 (1929).

' Langley, S. P., Ann. Astrophys. Obs., 1, table 23 (1900).

\section{Composition and Constitution of Ethylenebiguanide}

IN a previous note on "A New Type of Complex Silver Compounds with Tervalent Silver"', the com. position of ethylenebiguanide was represented as that given by Dittler ${ }^{2}$ as. well as by Dübsky and coworkers ${ }^{3}$. This is, however, not correct. It has now been found that the so-called ethylenebiguanide, as prepared by us following the method described by Dittler and also by Dübsky and co-workers, is really an ethylene dibiguanide, $\operatorname{En}(\mathrm{BigH})_{2}$, of the composition $\mathrm{C}_{6} \mathrm{~N}_{10} \mathrm{H}_{16}$. It behaves as a quadridentate molecule with four points of attachment to the central silver atom instead of being bidentate like the so-called ethylenebiguanide. This difference was not detected before, as we had no reason to suspect the composition of the compound and satisfied ourselves only with the estimation of nitrogen and sulphate in a sample of the substance, which, however, agreed with those given by Dübsky and co-workers.' A subsequent estimation of carbon and water both in the substance under discussion and in its complex silver salt revealed this difference; $2 \mathrm{C}_{4} \mathrm{~N}_{6} \mathrm{H}_{9} \cdot \mathrm{H}_{2} \mathrm{SO}_{4}$ gives almost the same values for nitrogen and sulphate as $\mathrm{C}_{6} \mathrm{~N}_{10} \mathrm{H}_{16} 2 \mathrm{H}_{2} \mathrm{SO}_{4} .1 \cdot 5 \mathrm{H}_{2} \mathrm{O}$.

Therefore, the constitution of the tervalent silver complex should now be represented as : $\left[\mathrm{Ag}^{111} \mathrm{En}(\mathrm{BigH})_{2}\right] X_{3}$, 